\title{
Poliovirus antigenic sites and vaccines
}

SIR - Two recent News and Views commentaries have discussed the identification of a neutralizing antibodyinducing site on poliovirus ${ }^{1}$ and the possibility of using corresponding, synthetic peptides as vaccines ${ }^{2}$. Some conclusions drawn and definitions used in these articles differ from our view of the subject matter.

In the first commentary, the author explains how recent studies ${ }^{3,4}$ on poliovirus type 3 (PV3) have identified an amino acid sequence of the viral structural protein VP1 which "represents the site on the virus involved in virus neutralization". The implication that only a single antigenic site is involved in the induction of neutralizing antibodies is probably premature for PV-3. It is certainly incorrect for poliovirus, type 1 (PV-1) $)^{5-7}$.

For clarity, we will use the following definitions and abbreviations. The term neutralization antigenic site $(\mathrm{N}-\mathrm{Ag})$ of a virus describes that region of a capsid protein, composed of contiguous (or noncontiguous) amino acids, that induces and binds neutralizing antibodies. A neutralization epitope (N-Ep) is the molecular conformation of a sequence of contiguous (or non-contiguous) amino acids of the $\mathrm{N}-\mathrm{Ag}$ that a neutralizing monoclonal antibody (N-mcAb) will recognize and bind. A single $\mathrm{N}-\mathrm{Ag}$ may be capable of expressing many $\mathrm{N}-\mathrm{Eps}$. The $\mathrm{N}$-Eps in such a cluster may or may not overlap functionally, that is, genetic variants of a virus resistant to neutralization by one $\mathrm{N}$-mcAb may or may not escape neutralization by other $\mathrm{N}$-mcAbs of the same cluster $^{5,6}$.

P. Minor and D. Evans and their colleagues elicited N-mcAbs against PV-3 that recognized different N-Eps. Surprisingly, all of these N-Eps belong to a single N-Ag on capsid protein VP1, as shown by genetic and biochemical methods ${ }^{3,4}$. With PV-1 we have used a similar approach ${ }^{6,7}$. Two N-Ags on capsid protein VP1 were identified by a binding assay (ELISA) of groups of N-mcAbs with two different virus-specific, synthetic peptides. Each group of N-mcAbs recognized a different $\mathrm{N}-\mathrm{Ep}$ as shown by genetic analyses ${ }^{6}$. A third N-Ag on VP1 was identified by the ability of a third peptide, itself unable to bind to any $\mathrm{N}$-mcAbs available to us, to "prime" a neutralizing immune response". Two other $\mathrm{N}$-Ags exist, one each on the VP2 and VP3 structural proteins of $\mathrm{PV}-1^{5,8,9}$. In the case of VP2 the exact amino acid sequence involved is known'.

PV-1 therefore has at least five different $\mathrm{N}$-Ags. This does not mean, however, that every animal inoculated with poliovirus will produce antibodies to all of these sites. We believe that two of the N-Ags of PV-1 are more readily recognized than the others by the animal's immune system, a phenomenon that would explain why $\mathrm{N}$-mcAbs can generally be elicited only against these two "immunodominant" sites $^{7}$. But it should be kept in mind that the current assignment of dominant N-Ags is solely based upon results obtained with $\mathrm{N}$-mcAbs. We suggest that the $\mathrm{N}-\mathrm{Ag}$ identified for PV-3 (ref.3) is such an immunodominant site, but we predict that PV-3 has several other neutralization sites that are recognized to a lesser degree by the immune system.

Neutralizing antibodies may destroy the infectivity of the poliovirion by different mechanisms. The $\mathrm{N}$-mcAbs directed against capsid polypeptide VP1 alter the isoelectric focusing point (pI) of $\mathrm{PV}-1^{6}$. Neutralization requires bivalent binding ${ }^{10,11}$. This is in contrast to neutralizing antibodies directed against capsid polypeptide VP3 that do not induce a change of $\mathrm{pl}$ and that do not require bivalent binding for inactivation ${ }^{5,6,10}$. In view of all of this, which peptides should be selected and from which regions of the capsid proteins should they originate in order to construct a synthetic vaccine?

We have tested so far 6 peptides, 5 corresponding to sequences of capsid protein $\mathrm{VP}^{7}$ and one to capsid protein $\mathrm{VP}^{9}$, for their ability to elicit a neutralizing antibody response. Success was scored only with a peptide corresponding to the amino acid sequence of one of the immunodominant $\mathrm{N}$-Ags of VP1 and with a peptide corresponding to an amino acid sequence of VP2. Does this result imply that these peptides are the only ones "whose further potential as a peptide vaccine is worth exploring" 2 ?

No rules have been found that can predict whether or not a peptide will mimic the N-Eps of a N-Ag. The final selection of the sequence of a peptide and its chain length must still be determined empirically. But even if a peptide carrying the sequence of a $\mathrm{N}-\mathrm{Ag}$ is unable to elicit neutralizing antibodies, it can be "seen" and memorized by the immune system of the

\section{Availability of Mo and HTLV-II}

SIR - Following recent publications, I have had numerous inquiries regarding the availability of the Mo B-lymphoblast line - an Epstein-Barr virus-transformed cell line from the patient (Mo) which is productively infected with HTLV-II - and of HTLV-II and lymphokine producing cell, derived by transforming normal $\mathrm{T}$ lymphocytes with HTLV-II ${ }^{1}$. The Mo-B line and various HTLV-II-transformed T-cell lines are available upon reasonable request. So too are HTLV-II molecular probes ${ }^{2}$, HTLV-I-infected primary cells (ME) and HTLV-I-infected transformants which produce lymphokines ${ }^{3}$.

I should also like to mention the position regarding the original Mo T-lymphoblast line, first isolated in 1978 and subsequently restricted because of a University of California agreement stipulating that the cell test animal. We have shown recently that the recall of this immunological memory to a peptide can lead to a powerful "priming" effect of a neutralizing immune response ${ }^{7}$.

Some viruses, such as foot-and-mouth disease virus (an aphthovirus belonging to the same family as poliovirus) and influenza virus, can tolerate the rapid genetic change of their dominant $\mathrm{N}$-Ags without loss of viability. This phenomenon is highly advantageous for a virus as it distracts the attention of the immune system from other, less variable N-Ags, and leads to variants which are resistant to neutralization. "Priming" of a neutralizing immune response with peptides, we speculate, may be useful in alerting the immune system not only to dominant but also less dominant (and possibly less variable) N-Ags, which, in turn, may produce long-term protection against viruses undergoing antigenic drifts or shifts.

ECKARD WIMMER

BRADFORD A. JAMESON

Department of Microbiology,

School of Medicine,

State University of New York at Stony Brook,

Stony Brook, New York 11794, USA EMILIO A. EMINI

Division of Virus and Cell Biology, Merck, Sharp and Dohme

Research Laboratories,

West Point, Pennsylvania 19486, USA

1. Brown, F. Nature 304, 395-396 (1983)

2. Newmark, P. Nature 305,9 (1983)

3. Minor, P.D. et al. Nature 305, 674-679 (1983)

4. Evans, D.M.A., Minor, P.D., Schild, G.C. \& Almond, J.W. Nature 304, 459-462 (1983)

Ernini, E.A., Dorner, A.J., Dorner, L.F., Jameson, B.A. \& Wimmer, E. Virology 124, 144-151 (1983).

6. Emini, E.A., Kao, S.-Y., Lewis, A.J., Crainic, R. \& Wimmer, E. J. Virol. 46, 466-474 (1983)

7. Emini, E.A., Jameson, B.A. \& Wimmer, E. Nature 304 699-703 (1983).

8. van Wezel, A.L., van der Marel, P., Hazendonk, T.G. Boer-Bak, V. Henneke, M.A.C. J. biol. Standard (in the press).

9. Emini, E.A., Jameson, B.A. \& Wimmer, E. in Modern Approaches to Veccines (eds Chanock, R. \& Lerner, R. (Cold Spring Harbor Press, New York, in the press). 10. Emini, E.A., Ostapchuk, P. \& Wimmer, E. J. Virol. 48, 547-550 (1983)

1. Icenogle, J. et al. Virology 127, 412-425 (1983).

line would not be made generally available until a patent is issued to the university. A patent on the cell line will be issued before 14 April 1984 and the cell line will then be available from the American Type Tissue Culture Collection, 12301 Parklawn Drive, Rockville, Maryland 20852.

The problem of availability of biological reagents is a complex and important issue. Certainly, biological materials should be available to the scientific community for research purposes; however, the availability of these materials for commercial purposes needs to be regulated. Many factors enter into the equation of how biological reagents are made available. The "ownership" question needs to be established even if the transaction is between nonprofit organizations. Guidelines related to the exchange of reagents 